\title{
Coherent Light Weight Planning for Secure Cloud Computing
}

\author{
Putta srivani, Dasari Madhavi, R.Sridevi
}

\begin{abstract}
A significant test in cloud and versatile distributed computing is to guarantee security and protection of client's data (e.g., money related information, wellbeing record, area data) from attacks. It is significant for a cloud service provider (CSP)) to build up trust and addition certainty by providing proper security and privacy to the clients.. Validation is significant for setting up responsibility and approval of the clients while distributing cloud resources. Researchers have proposed a few procedures, for example, token-based, picture and biometric based, to make the verification procedure progressively productive, secure and easy to understand. In this paper, talk about various confirmation methods proposed for both cloud and versatile distributed computing situations. This paper arranges the calculations dependent on its information, for example the credentials required for approving clients. Notwithstanding, this work emphasize that the arrangement isn't exact, as it is hard to group the validation calculations depending on more than one client credentials. To comprehend the intricacy and postponement of a validation procedure, the proposed work center around the quantity of elements associated with a confirmation procedure and the quantity of handshakes occurring between them. This paper additionally analyze the confirmation calculations based on plan standards and security faults.
\end{abstract}

Index Terms--Could Computing, Mobile Cloud computing, Authentication, Confidentiality, Infrastructure-as-a-Service,

\section{INTRODUCTION}

With the advancement about dispersed enrolling and the inevitability from ensuring smart Mobile phones, people are controlled getting to know in this manner period from stating data allowing model verified near to which those data will be put out in the cloud and the Mobile phones are used should store/recover those piece of data starting with that cloud. Normally, Mobile phones hardly bring obliged limit room and enrolling essentialness. Indeed, those cloud data require a tremendous extent of inclinations. In such a situation, to fulfil the sensibility execution, it is essential to use those focal points suited toward those cloud expert concentration to store and offer those a lot of the data [1].

These days, separate cloud worthwhile requests bring been commonly used. In these applications, people (information owners) can exchange their photographs, reports and grouped records of the cloud and What's progressively offer this data for different people (information clients) they the hop and in the open entryway with giving [2].

Revised Manuscript Received on April 25, 2020.

* Correspondence Author

Dr. Putta srivani*, Professor, Department of CSE, Sridevi Women's Engineering College, Telangana

Dr. Dasari Madhavi , Professor, Department of CSE, Sridevi Womens Engineering College, Telangana

Dr.R.Sridevi, Professor \& HOD, Department of CSE, JNTUHCEH, Telangana

(c) The Authors. Published by Blue Eyes Intelligence Engineering and Sciences Publication (BEIESP). This is an open access article under the CC BY-NC-ND license (http://creativecommons.org/licenses/by-nc-nd/4.0/)
CSPs also suit data affiliation comfort with the greater part of the data owners. Since singular data reports need support sensitive, the greater part of the data owners need support license up to make their data records open on other hand must be met with all the data customers.

Unquestionably, data security of the individual problematic a lot of the data is an authentic stress for the right bigger piece of data owners [3]. Those best on people advantage gain with power frameworks offered Toward the CSP may be whichever not sufficient then again not unimaginably made. They can't help each server among the prerequisites of a large portion of the data owners. In any case, at cloud exchange their prevailing piece of data records onto the cloud, they are expelling the greater part of the data finished a part the value is out of their control, and the CSP may stay with an eye once customer a lot of the data for its business great conditions and besides one of a kind motivations. Second, people need on sending those question verbalizations to each data customer on the off condition that they essentially need will assignment those encoded a lot of the data for explicit clients, which will be tremendously messing up [4]. To streamline those reductions association, the vast majority of the data owner may isolate a lot of the data customers under different gettogethers and send the watchword of the gatherings which they require on allocating those bigger piece of data. Be that in like way, it may, this framework obliges fine-grained gain opportunity to power. In the two cases, the secure key affiliation may be a fundamental issue [5].

Distributed computing likewise at times known as utility processing empowers advantageous and on-request arrange access to countless registering assets, for example, organize, programming, applications and administrations from anyplace over the globe with communication of expert. It has gotten irreplaceable in the readiness of many cloud foundations [1]. The fundamental guideline of distributed computing is to utilize the virtualization innovation to offer the ideal types of assistance from imparted equipment to the point of making a greatly improved utilization of circulated cloud data. The distributed computing joins many existing innovations and framework comprising of PCs, dispersed applications, data and system assets. Distributed computing is commonly classified into three cloud administration models, in particular Software as a Service (SaaS), Platform as a Service (PaaS) and Infrastructure as a Service (IaaS) [2]. These cloud models are portrayed in Fig. 1. In Software as a Service (SaaS) model, the end clients use the applications running on cloud foundation from cell phones or programs on a rental or per-use premise. Run of the this models incorporate Microsoft Office 365, Google applications, Salesforce.com, and so on.

Blue Eyes Intelligence Engineering

\& Sciences Publication

DOI: 10.35940/ijeat.D9137.049420

Journal Website: www.ijeat.org 
In Platform as a Service (PaaS) model, the administrations like center working framework and building square administrations are caused accessible to end clients so they can run their applications. Models right now are Windows Azure, Google App Engine, Force.com, and so forth. In Infrastructure as a Service (IaaS) model, the cloud sellers offer segments of foundation like systems, processor, memory, virtualization, and so forth to the end clients at a particular time and cost on a concurred premise. Instances of IaaS incorporate Amazon EC2, Microsoft Azure Platform, Cisco Metapod, and so on.

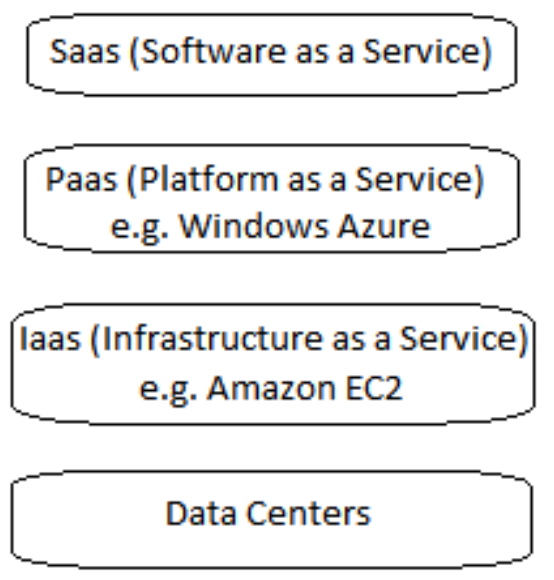

Fig. 1. Different cloud service models

Server farms establish the framework of distributed computing. These for the most part comprise of thousands of servers which are associated with one another and are worked in less involved regions [3-5]. coherent light weight planning in a way that permits the services to be accomplished utilizing better assistance level understanding, and least vitality to meet nature of Infrastructure as a Service. For this reason, an improved developmental calculation has been structured and executed. The security of the framework has been provisioned utilizing Fast Encryption method on pictures and content information. The paper is sorted out as follows. Segment II gives a diagram of the related work did toward cloud power utilization and situation. Segment III shows the design of the proposed work. In Section IV, the proposed strategy has been examined. Area $\mathrm{V}$ exhibits the outcomes and conversation.

\section{PREVIOUS WORKS}

A few systems have been proposed in past to deliver the issues to quantify the force utilization of each cloud service and verifying the information move across cloud. A portion of these systems proposed by various creators have been plot beneath:

Wang et al. considered the issue of guaranteeing trustworthiness of information put away on cloud servers. The creators accomplished information uprightness while permitting both open certainty of information through outsider inspector and dynamic information tasks. Yu et al. tended to the security challenges for information security and access control by joining access approaches which depended on information traits and permitting end clients to appoint the computational overheads engaged with get to control to untrusted servers. The methodologies of trait based encryption, lethargic re-encryption and intermediary re-encryption were investigated and consolidated in a one of
The focal point of this paper is on structuring the

a kind way. The proposed plan end up being profoundly secure and proficient under winning security models. Subashini et al. did an overview of various security dangers pervasive in the cloud administration models. Researchers underlined that however there were various points of interest in utilizing a cloud engineering, yet, the issues like security, power utilization, precision, and so on, stay uncertain. Beloglazov et al. proposed the cloud data asset allotment calculations using the dynamic solidification of virtual machines. The engineering standards for cloud data administration, data distribution approaches and booking calculations were advanced remembering the ideal nature of administration parameters and force utilization highlights of changing models. The outcomes uncovered that in contrast with static asset assignment procedures, the proposed strategy productively decreased data utilization in cloud server. $\mathrm{Li}$ et al. portrayed that inferable from heterogeneous nature of occupations, distinctive cloud services have diverse requirement times even on same physical machine. Besides, the physical machines are heterogeneous in nature. In this manner, cloud service situation times on them likewise change. Researchers recommended a disconnected cloud service arrangement plan utilizing copied cloud service data movement and an on-line cloud service situation technique through a genuine cloud service relocation process. The outcomes approved that the proposed calculation was profoundly proficient in situation of cloud services. Zhang et al. actualized and sent cloud service data to address the issue looked by cloud specialist organizations in provisioning a few cloud services to meet end clients' registering needs. The cloud service innovation is another cloud service provisioning apparatus which depends on the perception that when a visitor cloud service begins, the information got to by the cloud service follow. Additionally, a similar sort of cloud services recovers practically identical information obstructs during the booting procedure paying little heed to their arrangement settings. The test results demonstrated that cloud service end up being amazing cloud service provisioning instrument regarding idleness, adaptability, and I/O execution for IaaS cloud. $\mathrm{Gu}$ et al. proposed a cloud service power metering based method for estimating the data by cloud services while they were going after assets on a similar server. The technique recursively split the information dataset into two subsets relying upon the chosen asset highlight, for example, CPU, store, and so forth. The proposed technique end up being fitting for continuous utilize and very compelling force the board device for cloud service server. The outcomes demonstrated that the exactness of the technique was about $98 \%$ for various sorts of uses running in cloud services. Li et al. depicted that cloud service distribution issue for numerous occupants in cloud server farms is a NP difficult issue. Researchers proposed a layered dynamic asset portion calculation dependent on the numerous issues. The calculation made the minimization of the total of the cloud service system distances across of all inhabitants as improvement objective and to bring down the asset discontinuity in cloud condition, diminished the distinctions in the Quality-of-Service (QoS) among various occupants and upgraded the general Quality-of-Service.
Published By:

Blue Eyes Intelligence Engineering

\& Sciences Publication

(C) Copyright: All rights reserved. 
The outcomes unmistakably demonstrated that the proposed calculation proficiently settled the cloud service asset distribution issue in cloud server farms. Chowdhury et al. planned and executed different cloud service arrangement calculations to take care of the issue of utilization and execution necessities for meeting the nature of Infrastructure-as-a Service. Researchers utilized combination of virtual machines dependent on past asset use information. Both low level and high level has been distinguished and different arrangements were proposed to address the issue of situation of different cloud service examples on a host machine for using the asset and expanding the arrival. The after effects of exhibited that the proposed strategies, particularly, the altered most noticeably terrible fit diminishing cloud service position approach performed altogether superior to the current virtual machine situation calculation structured in CloudSim. Dong et al. acquainted avaricious undertaking scheduler with explore an effective planning for cloud server. The undertaking task was defined as number programming issue to lessen the utilization by cloud information servers by proposing the most-proficient server-first assignment planning. Results demonstrated that the utilization of the proposed planning was multiple times lesser than the one dependent on irregular based previous plan.

\section{PROPOSED WORK}

Aside from number of arrangements that have been advanced to lessen power utilization for cloud server and improve administration level understanding, the proposed approach accept that the IaaS cloud has hubs which are grouped to make branches. For every hub, there is a related uncommon programming bundle known as virtualization whose primary activity is to make and keep up the cloud server virtual machines. Moreover, it satisfies the end clients for getting to the ideal assets by empowering the segregation and deliberation of fundamental equipment and low-level functionalities in the cloud. There is a lot of end client undertakings every one of which may have a few subtasks. A subtask is dispensed to an asset at once and assets are accessible constantly. The point is to expel the virtual machines from under-loaded has so they can be closed to spare the power and diminish the operational expense. It is likewise attractive to relocate the virtual machines from over-laded has with the goal that isn't corrupted. To achieve this, an improved Evolutionary Algorithm has been utilized because of its ground-breaking search ability, worldwide arrangement discovering highlight and smaller structure with barely any parameters [16-17].

The flow graph for the proposed work is shown in Fig. 2. First, the virtual machines are placed on the host after verifying the resource vectors of both host as well as the virtual machines.

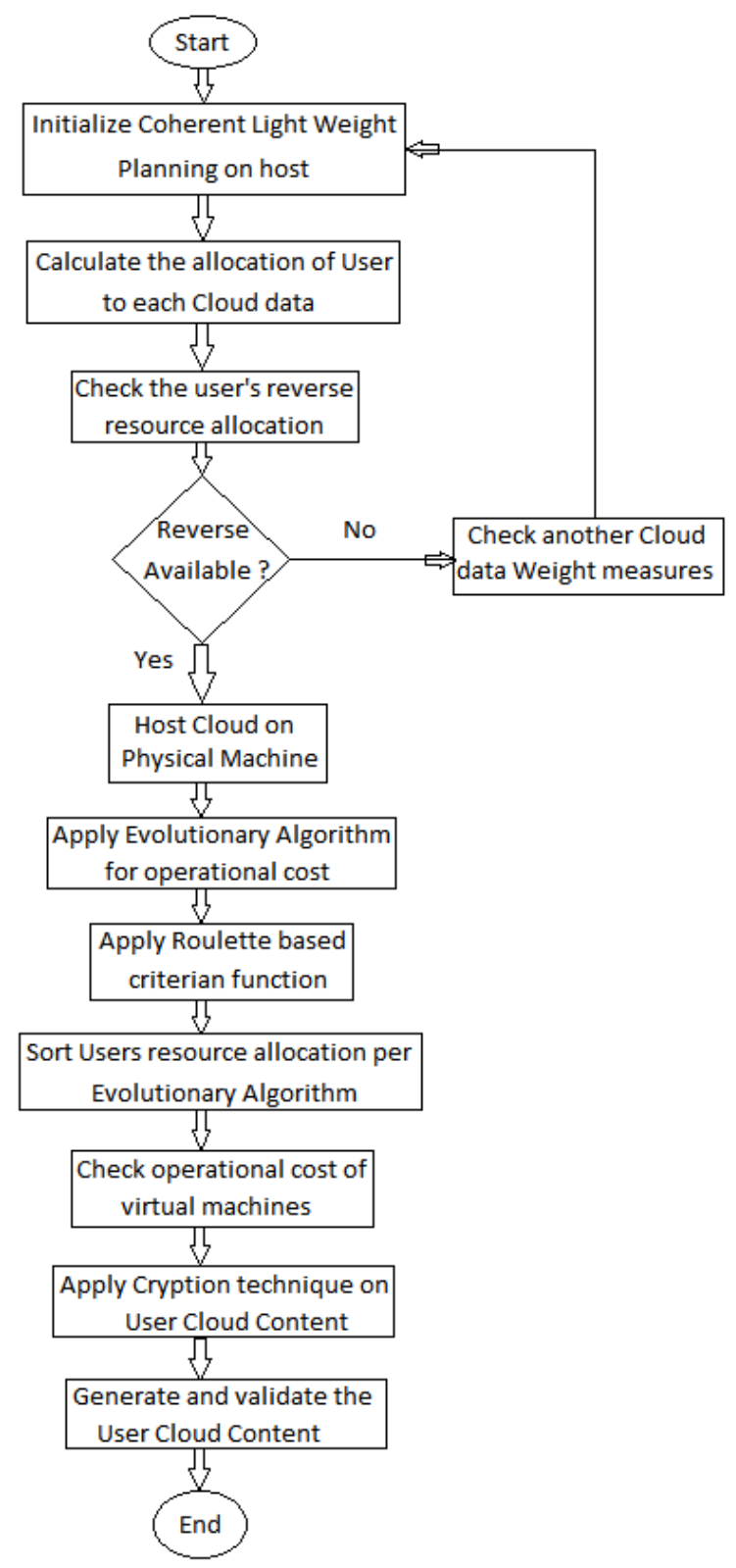

Fig. 2. Flowchart of the proposed work

On the off chance that satisfactory assets are accessible on the host, at that point VM will be put on that host. After VM situation, the errand distribution on the VM is finished. The point is to diminish the operational expense exhausted for task of undertakings on VM. To achieve this, proposed Evolutionary Algorithm is actualized which sorts the undertakings and spots them on the reasonable VM concurring the Roullet based model capacity. Therefore, the operational expense is determined. In the way of applying Evolutionary calculation, the security is imagined utilizing Fast Encryption Algorithm to shield the information. Next, the outcomes are created and contrasted with existing calculation [14] to prove the proposed research.

There are eight host machines on which numerous virtual machines can be conveyed. The total list is put away on the virtual machines and activities are performed in like manner. The encryption is done on the content and the twofold information. The proposed calculation works in following stages:

Published By:

Blue Eyes Intelligence Engineering

\& Sciences Publication

(C) Copyright: All rights reserved. 
a. Initially, the virtual machines are out of state and no task is allotted to them.

b. Subsequently, the list is encouraged and the overload condition is appeared. At whatever point a machine is in the over-load condition, the shade of that machine changes to red. The edge an incentive for over-heating is set to $80 \%$ of CPU usage.

c. The security is given utilizing Fast Encryption Algorithm (FEAL) which is an augmentation of DES encryption calculation. It is applied to both plain content and double information.

d. Several security threats, are performed on cloud to check on the off chance that it is protected against threats or not.

e. After the encryption of information on the cloud, the unscrambling procedure becomes an integral factor. The plain content is unscrambled to a meaningful arrangement. It is seen that the scrambled content is able enough against the threats on security.

f. After all the final tasks on the plain content, the encryption and unscrambling of parallel information, i.e., pictures are performed. The pictures are subdivided into squares and afterward, square figures are delivered and transmitted across cloud.

g. The calculation likewise shows the area of the particular hub on which the record is scrambled and is put away along these lines.

h. Finally, the outcomes are produced, gathered and approved utilizing four quantitative measurements, to be specific, CPU usage, power use, effectiveness and over use.

In this proposed work, structure data is encoded before moving to the cloud. Mix of Attribute Based Encryption and Byte Rotation Encryption Algorithm are used for the encryption of the data. ABE will recognize the attributes of the data and BREA will perform arrange things on the square of the data to be encoded. In the way of performing encryption task, an unpredictable key is created near to the encoded data. Data will be send in mixed association to specific customer. To interpret this data gatherer needs to enter the One Time Password (OTP) which will be facilitated with key made using ABE count.

\subsection{Proposed System Algorithm}

\section{Step-1: Start}

Step-2: Accept data from the customer.

Step-3: The Attributes of the data from the customers' task plans are gotten by the Attribute-Based Encryption.

Step-4: With the help of these Attributes, Random Key is delivered, and kind of data is gotten for encryption by BRE count.

Step-5: The data is changed over into proportional number of squares and $\mathrm{N} x \mathrm{~N}$ system will be delivered dependent on these squares.

Step-6: Based on no. of squares, pool of strings will be made.

Step-7: Run the strings in multi focus structure to make encoded data in short proportion of time.

Step-8: A encrypted data enter is created with the ultimate objective to open the encoded report which is taken care of in the cloud.

Step-9: The encrypted key is shared to the customer by methods for email or versatile number of the endorsed customer. This key will be used to translate the encoded report.
Step-10: The record taken will be decoded in the primary casing using the key.

Step-11: Stop.

\section{COMPARATIVE RESULTS AND DISCUSSIONS}

The proposed calculation is executed in the Java Web Application and NetBeans, which is a distributed computing reproduction toolbox. The outcomes are created and contrasted and the Modified Worst Fit Decreasing Virtual Machine Placement (MWFDVP) approach as far as force usage, CPU use, effectiveness and over-use. Table. 1 and Table. 2 show power utilization and CPU usage individually, utilizing MWFDVP and proposed approaches. The cloudlet size parameter is taken on n-hub which is expanded up to 80 to look at the adaptability of the methodology.

Table 1. Power Consumption vs Cloudlet Size for [18], [27] and proposed approaches

\begin{tabular}{|c|c|c|c|}
\hline & \multicolumn{3}{|c|}{ Power Consumption } \\
\hline Cloudlet Size & [18] & {$[27]$} & Proposed \\
\hline 0 & 0 & 0 & 0 \\
\hline 5 & 0 & 0 & 0 \\
\hline 10 & 90 & 110 & 121 \\
\hline 15 & 92 & 115 & 130 \\
\hline 20 & 93 & 121 & 135 \\
\hline 25 & 94 & 123 & 137 \\
\hline 30 & 95 & 125 & 139 \\
\hline 35 & 95 & 127 & 141 \\
\hline 40 & 96 & 128 & 145 \\
\hline 45 & 97 & 128.5 & 145.5 \\
\hline 50 & 97 & 128.5 & 146 \\
\hline 55 & 97.5 & 130 & 150 \\
\hline 60 & 97.5 & 131 & 152 \\
\hline 65 & 98 & 132 & 155 \\
\hline 70 & 98.5 & 133 & 159 \\
\hline 75 & 99 & 135 & 160 \\
\hline 80 & 100 & 137 & 162 \\
\hline
\end{tabular}

Table 2. CPU Utilization versus Cloudlet Size for [18], [27] and proposed approaches

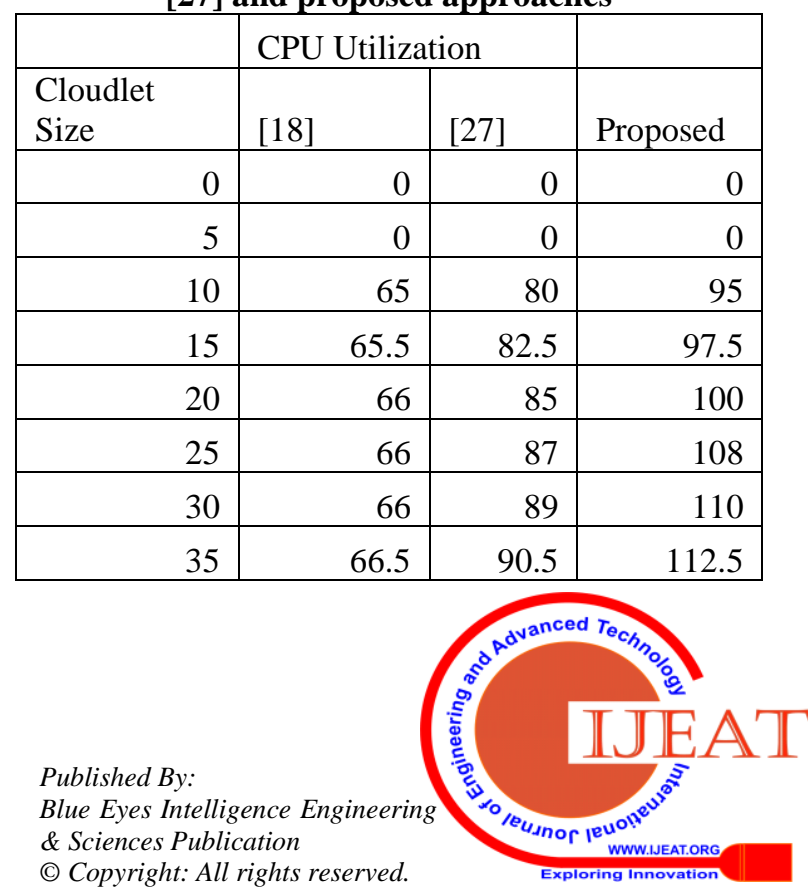




\begin{tabular}{|r|r|r|r|}
\hline 40 & 67 & 92 & 114 \\
\hline 45 & 67.5 & 93 & 115 \\
\hline 50 & 68 & 93.5 & 115.5 \\
\hline 55 & 69 & 95 & 117 \\
\hline 60 & 70 & 96 & 118 \\
\hline 65 & 72.5 & 96 & 118 \\
\hline 70 & 75 & 96.5 & 118.5 \\
\hline 75 & 75.5 & 97.5 & 119.5 \\
\hline 80 & 76 & 98 & 120 \\
\hline
\end{tabular}

Both the tables (Table 1 and 2 ) obviously shows that the utilization of proposed work essentially diminishes power utilization and CPU use in contrast with the Modified Worst Fit Decreasing Virtual Machine Placement (MWFDVP) approach. Table 3 shows how the proficiency of data is expanded utilizing the proposed approach. On the other hand, the quantity of hosts are characterized which are expanded up to 5 to look at the adaptability of the methodology, the effectiveness parameter is characterized.

Table 3. Effectiveness versus Number of Hosts for [18], [27] and proposed approaches

\begin{tabular}{|r|l|l|l|}
\hline & \multicolumn{2}{|l|}{ Efficiency } & \\
\hline Host & \multicolumn{1}{|l|}{$[18]$} & {$[27]$} & Proposed \\
\hline 0 & 0 & 0 & \multicolumn{1}{l|}{0} \\
\hline 0.5 & 0 & 0 & 0 \\
\hline 1 & 95.125 & 98.125 & 101.125 \\
\hline 1.5 & 94.875 & 98.125 & 101.125 \\
\hline 2 & 94.6 & 98.125 & 101.05 \\
\hline 2.5 & 94.5 & 98 & 101 \\
\hline 3 & 94.25 & 97.8 & 100.5 \\
\hline 3.5 & 94.2 & 97.6 & 100.4 \\
\hline 4 & 94.125 & 97.5 & 100.2 \\
\hline 4.5 & 94.2 & 97.5 & 100.1 \\
\hline 5 & 94.1 & 97.48 & 99.5 \\
\hline
\end{tabular}

Table 4. shows how over use of difficulty vector utilizing proposed approach is decreased. On the other hand, the quantity of virtual machines are shown which is expanded up to 50 to look at the versatility of the methodology, while, on the other side, the over usage parameter is appeared.

Table 4 Over Utilization versus Cloudlet Size for [18], [27] and proposed approaches

\begin{tabular}{|c|c|c|c|}
\hline & Over Utiliza & ion & \\
\hline $\begin{array}{l}\text { Cloudlet } \\
\text { Size } \\
\end{array}$ & [18] & [27] & Proposed \\
\hline 0 & 0 & 0 & 0 \\
\hline 5 & 0 & 0 & 0 \\
\hline 10 & 3 & 7 & 11 \\
\hline 15 & 3.5 & 8 & 12.5 \\
\hline 20 & 4 & 9 & 14 \\
\hline 25 & 5.5 & 10 & 15.5 \\
\hline 30 & 7.2 & 11.2 & 15.8 \\
\hline 35 & 8.1 & 11.5 & 15.9 \\
\hline 40 & 9.1 & 12.2 & 16.4 \\
\hline
\end{tabular}

\begin{tabular}{|r|r|r|r|}
\hline 45 & 10.1 & 13.2 & 17.3 \\
\hline 50 & 11 & 14 & 18 \\
\hline
\end{tabular}

Here the use of Coherent Light Weight Planning Attribute Based Encryption (CLWPABE) to scramble EHRs considering restorative organizations suppliers' characteristics or abilities, to unscramble EHRs, they should have the strategy of qualities required for appropriate access. The requirements and utilization of a cloud-construct EHR framework by considering CLWPABE is performed and showed, near to key assessments to take as a requirement at the adaptability and flexibility for proposed procedure.

\section{CONCLUSIONS}

Here, various assessments on get to control in cloud are totally established on quality based encryption count (ABE). Nevertheless, regular ABE isn't sensible for flexible cloud since it is computationally genuine and mobile phones has limited resources. Right now, proposed Coherent Light Weight Planning (CLWP) is sustained to these issue. It displays a novel CLWPABE computation to move genuine estimation overhead from mobile phones onto middle person servers, consequently it can help in handling the sheltered data sharing issue for versatile cloud. The preliminary outcomes show expresses that CLWP can ensures data security in flexible cloud and reduce the overtrouble on customers' side in convenient cloud. In future work, this paper designed the better approaches to manage ensure data dependability. To moreover tap the ability of flexible cloud, and besides ensure how to do figure content replication over existing data sharing plans.

\section{REFERENCES}

1. S. Yu, C. Wang, K. Ren, and W. Lou, "Achieving secure, scalable, and fine- grained data access control in cloud computing", in IEEE proceedings of Infocom, pp. 1-9, March 2010.

2. W. T. Tsai, X. Sun, and J. Balasooriya, "Service-oriented cloud computing architecture," in IEEE Seventh International Conference on Information Technology: New Generations (ITNG), pp. 684-689, April 2010.

3. R. Buyya, R. Ranjan, and R. Calheiros, "Intercloud: Utility-oriented federation of cloud computing environments for scaling of application services," Algorithms and architectures for parallel processing, pp. 1331, 2010.

4. Yu S., Wang C., Ren K., et al. Attribute based data sharing with an attribute revocation. in: Proceeding of 5th International Symposium on Information, Computer and Communication Security (ASIACCS), New York, USA: ACM press, 2010.

5. Y. Wang, K. W. Wong, X. Liao, and G. Chen, "A new chaos-based fast image encryption algorithm", Applied soft computing, vol. 11, no. 1, pp. 514-522, 2011.

6. S. Subashini and V. Kavitha, "A survey on security issues in service delivery models of cloud computing", Journal of network and computer applications, vol. 34, no. 1, pp. 1-11, 2011.

7. Jia W, Zhu H, Cao Z, et al. SDSM: a secure data service mechanism in mobile cloud computing. in: Proceedings of 30th IEEE International Conference on Computer Communications. Shanghai, China: IEEE, pp. 1060- 1065, 2011.

8. Beloglazov, J. Abawajy, and R. Buyya, "Energy-aware resource allocation heuristics for efficient management of data centers for cloud computing", Future generation computer systems, vol. 28, no. 5, pp. 755-768, 2012.

9. Y. Jadeja and K. Modi, "Cloud computing-concepts, architecture and challenges," in IEEE International Conference on Computing, Electronics and Electrical Technologies (ICCEET), pp. 877-880, March 2012.

Published By:

Blue Eyes Intelligence Engineering

\& Sciences Publication

(C) Copyright: All rights reserved. 
10. Sahai, H. Seyalioglu, and B. Waters, "Dynamic credentials and ciphertext delegation for attribute-based on encryption," in Advance in the Cryptology-CRYPTO. Springer, 2012.

11. K. Dasgupta, B. Mandal, P. Dutta, J. K. Mandal, and S. Dam, "A genetic algorithm (GA) based load balancing strategy for cloud computing”, Procedia Technology, vol. 10, pp. 340-347, 2013

12. K. Li, H. Zheng, and J. Wu, "Migration-based virtual machine placement in cloud systems", in IEEE 2nd International Conference on Cloud Networking (CloudNet), pp. 83-90, November, 2013.

13. W. Kim, "Cloud computing architecture", International Journal of Web and Grid Services, vol. 9, no. 3, pp. 287-303, 2013.

14. H. T. Dinh, C. Lee, D. Niyato, and P. Wang, "A survey of mobile cloud computing: architecture, applications, and approaches", Wireless communications and mobile computing, vol. 13, no. 18, pp. 1587-1611, 2013

15. Kan Yang, XiaohuaJia, Bo Zhang, RuitaoXie: "DACMACS: Effective Data Access Control for Multiauthority Cloud Storage Systems". IEEE Transactions on Data Forensics and Security, Vol. 8, No. 11, pp.1790 1801, 2013.

16. Z. Zhang, Z. Li, K. Wu, D. Li, H. Li, Y. Peng, and X. Lu, "VMThunder: fast provisioning of large-scale virtual machine clusters", IEEE Transactions on Parallel and Distributed Systems, vol. 25, no. 12, pp. 3328-3338, 2014.

17. Z. Dong, N. Liu, and R. Rojas-Cessa, "Greedy scheduling of tasks with time constraints for energy-efficient cloud-computing data centers", Journal of Cloud Computing, vol. 4, no. 1, pp. 5, 2015

18. M. R. Chowdhury, M. R. Mahmud, and R. M. Rahman, "Implementation and performance analysis of various VM placement strategies in CloudSim", Journal of Cloud Computing, vol. 4, no. 1, pp. 20, 2015

19. J. Li, D. Li, Y. Ye, and X. Lu, "Efficient multi-tenant virtual machine allocation in cloud data centers", Tsinghua Science and Technology, vol. 20, no. 1, pp. 81- 89, 2015

20. C. Gu, P. Shi, S. Shi, H. Huang, and X. Jia, "A tree regression-based approach for VM power metering”, IEEE Access, vol. 3, pp. 610-621, 2015

21. Denisow, S. Zickau, F. Beierle, and A. K"upper, "Dynamic location information in attribute-based encryption schemes," in Proceeding of 9th International Conference for Next Generation Mobile Application, Services and Technologies (NGMAST 2015). IEEE, 2015.

22. L. Touati and Y. Challal, "Efficient cp-abe attribute/key management for iot applications," in Computer and Information Technology (CIT), IEEE International Conference on 2015.

23. Chandni Patel, SameerSingh Chauhan Bhavesh Pate, "A Data Security Framework for Mobile Cloud Computing", International Journal of Advanced Research in Computer and Communication EngineeringVol. 4, Issue 2, February 2015

24. H. Hong, Z. Sun. "An efficient and traceable KP-ABS scheme with untrusted attribute authority in cloud computing", JoCCASA, 5(2).pp.I $-8,2016$.

25. Priya Dudhale Pise,Dr. Nilesh J Uke,’Efficient Security Protocol for Sensitive Data Sharing on Cloud Platform" in IEEE 2017.

26. Shubham Chandugade, Prachi More. "Survey on Lightweight Secured Data Sharing Scheme for cloud computing", International Research Journal of Engineering and Technology (IRJET)-ISSN: 2395-0056 Volume: 04 Issue: 10 Oct 2017.

27. Anupama Gupta1, Vivek Aggarwal2*, Ramandeep Singh Dhillon3, "A Secured and Enhanced Energy Efficient Solution for Cloud Computing Environment", Advances in Computational Sciences and Technology, Volume 10, Number 8 (2017) pp. 2541-2553.

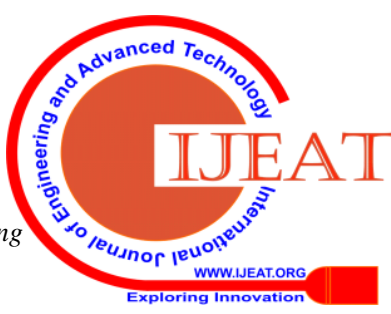

